Vol. 4, $n^{\circ} 1 \mid 2000$

Varia

\title{
La clémence du glaive : Plaider pour les criminels au siècle des Lumières à Genève
}

The Herman Diedericks Prize Essay for 1999 / Lauréate du prix Herman Diedericks 1999

Françoise Briegel

\section{OpenEdition}

Journals

Édition électronique

URL : https://journals.openedition.org/chs/846

DOI : $10.4000 /$ chs.846

ISSN : 1663-4837

Éditeur

Librairie Droz

Édition imprimée

Date de publication : 1 janvier 2000

Pagination : $9-29$

ISBN : 2-600-00433-5

ISSN : 1422-0857

Référence électronique

Françoise Briegel, «La clémence du glaive : Plaider pour les criminels au siècle des Lumières à

Genève », Crime, Histoire \& Sociétés / Crime, History \& Societies [En ligne], Vol. 4, $n^{\circ} 1$ | 2000, mis en ligne le 02 avril 2009, consulté le 22 mars 2022. URL : http://journals.openedition.org/chs/846 ; DOI https://doi.org/10.4000/chs.846 


\title{
The Herman Diedericks Prize Essay for 1999
}

\section{Lauréate du prix Herman Diedericks 1999}

\author{
La clémence du glaive: \\ Plaider pour les criminels au siècle des Lumières \\ à Genève
}

\author{
Françoise Briegel'
}

Si au niveau procédural, la justice criminelle genevoise du XVIII siècle est similaire, en beaucoup de points, à celle de la France, elle possède toutefois des caractéristiques qui prouvent sa modernité judiciaire, notamment la défense des accusés lors de leur procès. Dès 1734 , les prévenus, jugés en grand criminel, sont libres d'être défendus par un avocat, durant l'audience devant le Petit Conseil. Parallèlement à ce droit de défense, les prévenus peuvent, selon certaines conditions, recourir auprès du Conseil des CC (organe souverain en matière de grâce) contre la sentence définitive prononcée par le Petit Conseil. Ainsi, pendant et après le jugement, les accusés sont susceptibles d'obtenir, grâce à un avocat, la clémence des magistrats ou une modération de peine. Les défenseurs qui plaident devant les Conseils, opèrent selon une méthode argumentative déconstructive qui présente des analogies avec celle effectuée par la partie publique, lorsqu'elle doit qualifier les délits et requérir une peine contre les prévenus. L'étude des plaidoiries de défense et des recours en grâce met en évidence, notamment que les avocats sont largement influencés par les conceptions pénales des Réformateurs du droit au siècle des Lumières. Or, les magistrats chargés de rendre la sentence ou de se prononcer sur une commutation de peine sont-ils sensibles à ces arguments? Il s'avère que les plaidoyers ont une forte efficacité: environ une fois sur deux la sentence est modérée ou commuée. Déjà filtré par le Réquisitoire du Procureur général, l'arbitraire du tribunal criminel est donc limité d'une façon supplémentaire par les requêtes des défenseurs. Ainsi, la précocité du droit à la défense dans le système judiciaire de la République peut être lu comme l'un des symptômes du passage de l'État de justice à l'État de droit.

While criminal justice in 18th-century Geneva in many ways resembles the French system at the procedural level, a number of features nevertheless attest to the modernity of Geneva's justice system, especially when it comes to courtroom defense. As early as 1734, the accused in major criminal trials were free to be defended by a lawyer at the hearing before the "Petit Conseil». In parallel, the accused could, under certain conditions, appeal to

1 Françoise Briegel est assistante en histoire moderne à l'Université de Genève (Suisse). Elle prépare actuellement une thèse de doctorat, sur la place des avocats dans les procédures pénales, entre les $\mathrm{XVII}^{\mathrm{e}}$ et XVIII ${ }^{e}$ siècles dans la République de Genève, sous la direction de Michel Porret.

Cet article synthétise un mémoire de licence soutenu en février 1998 et dirigé par Michel Porret. 
the Council of the CC (sovereign in matters of pardon) against the final sentence handed down by the "Petit Conseil». It was thus possible for the accused, during and after the trial to obtain, with the help of a lawyer, either a pardon or a reduction of the sentence. The defenders pleading before the councils employed a deconstructive, argumentative method that has certain analogies with that used by the prosecution when defining the offense and demanding a punishment. A study of the speeches for the defense and the petitions for reprieve shows in particular that the lawyers were largely influenced by the way the Enlightenment reformers looked on crime. But did the judges responsible for handing down or commuting sentences appreciate such arguments? It turns out that the pleadings were highly effective: in nearly one out of two cases the sentence was reduced or commuted. Already filtered by the indictment presented by the prosecutor, the arbitrary action of the criminal courts was even further restricted by the presentation of the defense. The early appearance of the right to be defended in the judicial system of the Republic can thus be read as symptomatic of the passage from a state governed by the courts (État de justice) to the a state governed by law (État de droit).

$\mathrm{L}$ e renouvellement de l'historiographie de ces quinze dernières années ${ }^{2}$ a permis de rectifier l'image despotique de la justice d'Ancien Régime qui, déjà dès le milieu du XVIII ${ }^{\mathrm{e}}$ siècle, avait été portée devant l'opinion publique, par les Réformateurs et les philosophes. La représentation d'un accusé privé de tout droit, n'ayant pas connaissance des charges de l'accusation et subissant l'iniquité des juges, a été fortement remise en question. En effet, en France, même si l'Ordonnance criminelle de 1670 interdit aux accusés de crime grave d'être aidés par un conseil, Antoine Astaing ${ }^{3}$ a récemment montré que les doctrinaires n'y sont pas opposés. En outre, l'étude des procès met en évidence que les criminels peuvent être assistés par des avocats. La défense orale, en violation des ordonnances royales, reste parfois possible pour les accusés démunis et privés de famille, notamment lorsque ces affaires sont portées en appel ${ }^{4}$. De plus, les requêtes écrites (factums) sont autorisées. Les rédacteurs de ces mémoires sont souvent des avocats qui, malgré le secret de l'instruction, connaissent les pièces de la procédure, grâce à des greffiers complaisants. Le Parlement de Paris, chargé de juger en appel les criminels, transmet même les interrogatoires à la défense ${ }^{5}$. Cette pratique de la défense non légalisée existe également en Angleterre. En effet, dans les années 1720, les avocats commencent à intervenir au côté de l'avocat général (partie publique) et, en contrepartie, les juges autorisent les criminels à être assistés par des avocats. Cette pratique de la défense qui débute vers 1730 ne sera légalisée que cent ans plus tard ${ }^{6}$. Par ailleurs, en Allemagne, déjà dès le $\mathrm{XVI}^{\mathrm{e}}$ siècle, l'Ordonnance Caroline promulguée par Charles Quint en 1532, permet aux accusés d'être aidés par un conseil, tiré parmi les juges du tribunal (article 88) ${ }^{7}$. Dans les Pays-Bas, l'Ordonnance de Philippe II de 1570

\footnotetext{
Pour un aperçu de ces nouvelles perspectives de recherches voir Garnot (1998).

Astaing (1999).

Astaing (1999, p. 114).

Astaing (1999, pp. 280-294).

Beattie (1986, pp. 352-362).

Astaing (1999, p. 119).
} 
prévoit l'assistance d'un avocat ou procureur dans les affaires importantes et compliquées. Ainsi, dans la pratique et dans les textes de loi, il y a des accusés défendus par des avocats nommés par la cour (à l'exception des vagabonds et des voleurs de grand chemin). Cette pratique de la Flandre, reste toutefois tributaire des avis des Conseils provinciaux, elle n'est pas généralisée ${ }^{8}$. Au XVIII ${ }^{\mathrm{e}}$ siècle, dans les tribunaux de Savoie qui appliquent la même procédure que l'Italie, l'accusé a la liberté de choisir un avocat qui est tenu de l'aider. Les pièces de la procédure lui sont communiquées ${ }^{9}$.

Dans la République de Genève, la légalisation de la défense apparaît dès 1734 et, durant tout le XVIII ${ }^{\mathfrak{e}}$ siècle, sa pratique s'intensifie. Ainsi, dès le premier tiers du $\mathrm{XVIII}^{\mathrm{e}}$ siècle, les Édits qui forment le cadre légal genevois, autorisent les prévenus à prendre un avocat. Les défenseurs requièrent la clémence des magistrats par une méthode «déconstructive». C'est-à-dire, inversement à l'argumentation de la partie publique, ils désassemblent les preuves du délit, ils «requalifient » le crime en diminuant sa dangerosité et ils motivent la demande de clémence au moyen d'arguments juridiques ou philosophiques. Tout comme les Procureurs généraux, les avocats s'inscrivent dans l' «espace arbitraire» de la justice d'Ancien Régime, mais ils ne réclament pas, en écho aux Réformateurs du droit, un code pénal qui détermine la fixité des délits et des peines. Ils utilisent cette indétermination pour alléguer des faits et arguments juridiques favorables aux prévenus. À la pratique d'un arbitraire "constructif» de la partie publique ${ }^{10}$, se confronte alors l'arbitraire "déconstructif» des avocats. De plus, ces derniers, efficaces auprès des magistrats, possèdent un rôle «modérateur » dans le prononcé des peines. Leur intervention orale limite donc l'arbitraire des magistrats en matière de répression sévère de la criminalité grave.

\section{LE CADRE LÉGAL DE LA PROCÉDURE PÉNALE Á GENEVE (1734-1792)}

À Genève, le système pénal, pareillement à celui de la France, est inquisitoire (procédure secrète, écrite et non contradictoire). La République ne connaît, contrairement au royaume voisin, qu'un niveau de juridiction en matière de criminalité grave. Le Petit Conseil, organe politique et judiciaire, juge en dernier ressort toutes les causes traitées en grand criminel. Les membres du Petit Conseil (au nombre minimum de treize) se prononcent sur tous les crimes «atroces». Cette procédure démarre selon trois modalités: plainte, dénonciation ou à l'instance de la partie publique. Une fois l'information bouclée, les pièces sont transmises au Procureur général afin qu'il établisse son Réquisitoire et ses "Conclusions». La partie publique qualifie le crime selon des circonstances à charge ou à décharge et motive une peine qu'il requiert au Petit Conseil. Durant tout le XVIII ${ }^{\mathrm{e}}$ siècle, les sentences définitives rendues par le Petit Conseil sont arbitraires, dans le sens où les membres de ce Conseil ont la liberté totale dans le prononcé des peines, mais ces sentences suivent 9 fois sur 10 les "Conclusions » des Procureurs généraux ${ }^{11}$. Ces derniers, par une casuistique des circonstances du crime et des éléments biographiques du crimi-

\footnotetext{
8 Dupont-Bouchat (1997, pp. 245-246).

9 Sartoris (1773, Vol. 2, pp. 519-522).

10 Porret (1995).

11 Porret (1995, p. 101).
} 
nel, qualifient minutieusement les délits. Ils motivent les peines selon les doctrinaires du droit, la jurisprudence locale ou étrangère et le droit romain. La partie publique inscrit les sentences prononcées par le Petit Conseil dans un proto-légalisme. En conséquence, le pouvoir des magistrats à Genève peut alors être qualifié d'arbitraire «mitigé » ou «limité ».

En 1734, est instaurée à Genève la défense des criminels. Un avocat plaide pour un prévenu au moment du procès, avant que la sentence définitive ne soit prononcée: «Afin néanmoins que l'accusé puisse mieux se défendre, il lui sera donné, si lui ou quelqu'un d'autre pour lui le requiert un avocat et procureur à son choix pour faire dresser et proposer ses moyens de défense et exception [...] $\rangle^{12}$. En France, l'Ordonnance criminelle de Saint-Germain-en-Laye de 1670, en vigueur durant tout le XVIII ${ }^{e}$ siècle, interdit la défense des prévenus et stipule que: «[...] les accusés de quelque qualité qu'ils soient, seront tenus de répondre par leur bouche, sans le ministère de conseil qui ne pourra leur être donné même après la confrontation $[\ldots]{ }^{13}$. Dans le royaume voisin, même si la défense est parfois pratiquée durant le XVIII ${ }^{e}$ siècle, il faut attendre 1789 pour qu'elle soit légalisée ${ }^{14}$.

L'avocat qui rédige la plaidoirie dispose d'un délai de huit jours avant l'audience. Il peut consulter les pièces de la procédure (verbaux, témoignages, récolements, confrontations, expertises, etc.) à l'exception des «Conclusions » du Procureur général. Il n'est pas autorisé à divulguer les pièces consultées qui doivent rester secrètes, conformément au système inquisitoire. Finalement, le défenseur est obligé de les rendre au Secrétaire de Justice, une fois le jugement rendu.

A l'exception des contumax, tous les criminels détenus dans les prisons contre lesquels s'instruit une procédure extraordinaire ont la liberté de prendre un avocat pour établir leur plaidoirie. Ils ont le libre choix du défenseur qui est tenu de les défendre sous peine d'interdiction ${ }^{15}$. Dans les cas où les prévenus sont incapables de désigner un avocat et qu'ils souhaitent bénéficier de leur droit de défense, le Petit Conseil nomme d'office l'avocat le plus récemment immatriculé. Celui-ci propose ses services à l'accusé gratuitement ${ }^{16}$. Ainsi, la faculté d'être défendu est «[...] pure, simple, absolue; elle n'est point soumise à la détermination du juge, mais au seul arbitre du prévenu qui a le droit indistinctement de s'en prévaloir ou d'y renoncer ${ }^{17}$. Il n'y a aucune restriction légale qui limite la défense d'un prévenu. Bien au contraire, le Petit Conseil exerce un contrôle auprès des membres du Barreau afin que les droits à la défense des accusés soient respectés ${ }^{18}$. Ainsi, au moment du jugement, la lecture de la plaidoirie ouvre l'audience, puis l'avocat se retire et ensuite, les pièces de la procédure et les "Conclusions» de la partie publique sont lues. Sur cette base, le Petit Conseil prononce la sentence définitive.

Selon certaines conditions déterminées par la loi, les criminels sont autorisés à recourir contre leur sentence auprès du Conseil des Deux-Cents (dorénavant $\mathrm{CC}$ ).

12 Extrait du Registre du 14 juillet 1734, approuvé en Conseil général le 21 novembre 1734.

13 Article 8 du titre 14.

14 Article 10 du décret du 8 octobre-3 novembre 1789 de la Réformation provisoire de la procédure pénale.

15 Article 30 du Règlement de l'lllustre Médiation de 1738.

16 Bérenger (1801, p. 159).

17 Sartoris (1773, Vol. 2, p. 524)

18 L'avocat Ami Mallet en 1787, refuse successivement de défendre un voleur et un meurtrier, il sera suspendu de ses fonctions par le Petit Conseil, durant trois mois; voir Ms Hist. 110. 
Disposant du droit de grâce, ce Conseil, en usant de bonté et de clémence, possède depuis 1568 la compétence d'adoucir une sentence définitive. Motivés par leur pouvoir de pardon, les membres du CC choisissent de réduire ou de confirmer la peine du Petit Conseil. Une fois commuée, la peine est appliquée avec les mêmes formalités que pour une peine non adoucie. L'avocat ne dispose que de vingt-quatre heures pour établir le recours en grâce. En octobre 1784, une proposition, relative au recours en grâce et émanant d'un membre du Conseil des CC, est soumise au Petit Conseil. Si un accusé a refusé de présenter une plaidoirie, la suggestion stipule que soient octroyés «[...] à son avocat, huit jours pour dresser la requête de recours, bien entendu que le prévenu pourra abréger ce terme, s'il estime qu'un moindre nombre de jours lui suffit ${ }^{19}$. Cherchant à harmoniser les délais de consultation des pièces pour le recours et la plaidoirie, cette proposition est refusée par le Petit Conseil. Ce dernier préfère s'en tenir à l'usage en n'accordant que vingt-quatre heures de délai entre le jugement et le recours. «[...] Sans doute parce qu'il a senti combien il serait dangereux de laisser de l'intervalle entre la sentence du Conseil et le recours, surtout dans les cas où le prévenu tiendrait à des personnes accréditées, et où il aurait été condamné à une peine grave ${ }^{20}$. Ainsi, le délai de rédaction du recours reste de vingt-quatre heures. De plus, les «Conclusions» de la partie publique ne sont pas transmises au défenseur.

Le déroulement du recours est similaire à celui de la plaidoirie. Il ouvre la séance, puis les pièces de la procédure, les «Conclusions », et la sentence sont lues. Le CC se prononce enfin sur une modération de peine. Le Règlement de l'Illustre Médiation de 1738 stipule que le Conseil des CC «[...] décidera s'il y a lieu d'accorder grâce, ou de modérer la sentence, laquelle ne pourra être aggravée [...] ${ }^{21}$. La sentence criminelle, si elle n'a pas été portée en recours, est lue solennellement devant l'Hôtel de Ville en présence du peuple, juste avant sa mise à exécution. En cas de commutation de peine par le Conseil des CC, «[...] la sentence de grâce ou de modération de peine sera publiée avec la même formalité que les autres sentences afin que le peuple en sache les motifs ${ }^{22}$. Contrairement à ce que promet cet $\hat{E} d i t$ de 1714 , les motifs de commutation de peine ne sont jamais dévoilés et seul le paternel pardon du Conseil des $\mathrm{CC}$ accompagne la justification de la réduction de peine.

Le recours en grâce, en vigueur dans la République de Genève, n'est pas similaire avec l'appel pratiqué en France à la même époque. Si le Règlement de l'Illustre Médiation de 1738 précise que les sentences portées en recours devant le Conseil des CC ne peuvent être aggravées ${ }^{23}$, en France, au contraire, une sentence de juridiction inférieure portée en appel devant les Parlements peut être plus sévère. Ainsi, à Genève, les accusés qui sont autorisés à recourir ne sont pas jugés à nouveau par le Conseil des CC. Celui-ci utilise son pouvoir de grâce afin de commuer la peine. Par ailleurs, en France, l'appel est rendu obligatoire par l'Ordonnance criminelle de 1670 , lorsque les justices inférieures rendent une sentence qui «[...] porte condamnation de peine corporelle, de galères, de bannissement à perpétuité ou d'amende honorable soit qu'il y en ait appel ou non, l'accusé et son procès seront

19 Registre des Conseils du 25 octobre 1784, fol. 1219, dorénavant R.C.

$20 \quad$ R.C. du 25 octobre 1784, fol. 1219.

21 Article 31.

22 Article 22 des Édits civils de 1714.

23 Article 31 . 
envoyés ensemble et sûrement [...]» ${ }^{24}$ devant les cours. Contrairement à Genève, les Parlements français jugent donc à nouveau le criminel ayant fait appel. De ce fait, le recours en grâce pratiqué à Genève et l'appel en France ne sont pas assimilables.

Durant le XVIII ${ }^{e}$ siècle, tous les prévenus ne sont pas autorisés à demander grâce auprès du Conseil des CC. Les Édits civils de 1714, stipulent que seuls les Bourgeois et Citoyens ${ }^{25}$, condamnés au moins à un châtiment corporel peuvent recourir en grâce. En 1738, Le Règlement de l'Illustre Médiation, élargit pour la même peine, le droit de recours aux Habitants et Natifs. L'Édit du 11 mars 1768 permet à un plus grand nombre de criminels de demander grâce: «Aucun Citoyen, Bourgeois, Natif, Habitant, ou Sujet de la République ne pourra recourir au Conseil des Deux-Cents, s'il n'a été condamné par jugement définitif du Petit Conseil à une prison de six mois, à un bannissement d'un an, à la suspension des droits honorifiques de la Bourgeoisie pendant cinq ans, à être déclaré infâme en termes exprès; ou condamné à quelqu' autre peine plus grave que celles ci-dessus [...]» ${ }^{26}$. De plus, «les Criminels étrangers jouiront du même droit lorsqu'ils auront été condamnés à mort; \& le Conseil pourra les admettre à ce recours pour toute autre peine, lorsqu'il le jugera convenable $[\ldots]{ }^{27}$. Enfin, en 1791 , tous les Genevois, condamnés au minimum à une amende de 500 écus, peuvent être entendus par le Conseil des CC; les Étrangers gardant la faculté de recourir que s'ils ont été condamnés à la peine capitale. Le recours s'ouvre donc à une plus large population de criminels, puisque davantage de peines prononcées par le Petit Conseil sont susceptibles d'être portées devant le CC pour être adoucies ${ }^{28}$.

\section{LES SOURCES}

Grâce à un fond d'archives très bien conservées ${ }^{29}$, il est possible de quantifier le taux d'intervention des avocats dans les procès criminels des prévenus détenus dans les prisons pour crime grave ${ }^{30}$. Pour la période 1755-1791, sur 248 condamnations

24 Article 6, du Titre 26.

25 Les Citoyens jouissent de tous les droits politiques et économiques ainsi que de quelques avantages fiscaux. Les Bourgeois possèdent les mêmes droits économiques que les Citoyens, mais ne sont pas éligibles au Petit Conseil. Les Natifs (nés à Genève) ne possèdent aucun droit politique et ils n'ont pas accès à certains secteurs économiques prestigieux. Les Habitants possèdent un droit d'habitation qui les confinent dans les professions les plus basses. Les Sujets sont les habitants des territoires ruraux gouvernés sous le mode seigneurial. Les Étrangers résident temporairement en ville. Pour un développement concernant les différents statuts juridiques dans la République, voir Perrenoud (1979, pp. 182 et s).

26 Article IX, § II.

27 Article IX, § I.

28 La promulgation des Règlements ou Édits à Genève coïncide avec des troubles politiques (1738, 1768,1782 , etc.) qui font intervenir des médiateurs (Berne, Zurich, la France) afin de ramener la paix civile dans la République. Les revendications politiques des opposants au régime ont sans doute influencé les élites à entreprendre des réformes pénales qui permettaient à des couches sociales plus basses de bénéficier de droits plus étendus, notamment celui de la grâce.

Quelques 13500 procès et informations sont disponibles pour la période 1700-1792.

30 Pour une étude quantitative et qualitative de la criminalité réprimée à Genève au XVIII ${ }^{e}$ siècle, voir Porret, (1995, pp. 42 et s). 
prononcées en grand criminel ${ }^{31}, 75 \%$ des procédures contre des détenus ${ }^{32}$ ont été plaidées par un avocat (plaidoirie ou recours en grâce). Ce fort pourcentage d'intervention témoigne, pour la deuxième moitié du siècle, de la massive présence des juristes dans les procédures pénales. La justice genevoise d'Ancien Régime permet à des délinquants de bénéficier des services d'un professionnel du droit pour établir leur défense et leur recours. Cette pratique tend à se systématiser.

Comme je l'ai déjà évoqué, la plaidoirie s'adresse aux membres du Petit Conseil (autorité politique et judiciaire) qui est à Genève le tribunal suprême traitant les procédures pénales. Le recours, quant à lui, est présenté devant le Conseil des CC qui est le seul corps habilité à commuer les peines prononcées par le Petit Conseil. Les décisions de sanctionner plus ou moins sévèrement un accusé ou de commuer une peine prononcée n'appartiennent donc pas aux mêmes organes décisionnels. D'apparence lointaine, ces sources distinctes présentent pourtant des caractéristiques qui justifient une étude commune.

En effet, le cadre procédural ne présente aucune incompatibilité entre la plaidoirie et le recours, dans la mesure où un même prévenu peut faire plaider ses "défenses» et recourir au Conseil des CC. Il peut librement choisir un avocat pour défendre sa cause devant le Petit Conseil. Les textes de loi qui déterminent l'accès au recours sont plus restrictifs, mais ils ne stipulent nullement qu'un criminel n'a pas le droit d'être entendu devant le $\mathrm{CC}$, s'il a déjà présenté une plaidoirie.

$\mathrm{Au}$ cours du XVIII ${ }^{\mathrm{e}}$ siècle, les procès criminels, instruits pour crimes contre les individus, les biens, les mœurs ou l'État, témoignent de plus en plus de cas dans lesquels l'avocat intervient pour la plaidoirie et ensuite pour le recours. Lorsque l'avocat élabore successivement les deux requêtes pour le même prévenu, il calque très souvent les arguments contenus dans le recours sur ceux de la plaidoirie. Dans le procès instruit contre un jeune homme, accusé de meurtre en 1749, la présence des deux requêtes permet une comparaison précise ${ }^{33}$. Le premier document est structuré selon trois niveaux justificatifs; la jeunesse du suppliant, son éducation et les «circonstances» du crime. Ces arguments n'ayant aucun effet modérateur sur la décision des magistrats et pour tenter d'éviter la pendaison à l'accusé, le même avocat présente un recours à la grâce devant le Conseil des CC. Les mêmes arguments sont réutilisés et développés. Ici, le recours en grâce de l'avocat Bonnet est simplement un développement de la plaidoirie. Ainsi dans les procès contenant la plaidoirie et le recours, les approches argumentatives choisies par l'avocat sont similaires.

Le troisième élément qui justifie une approche conjointe des deux sources est relatif à la finalité des requêtes. La plaidoirie et le recours visent un objectif similaire: le prévenu jugé cherche dans les deux cas à éviter une peine trop lourde. Lors de la défense, l'avocat présente des arguments pour inciter les juges à la modération. Dans le recours, il tâche de persuader les magistrats de diminuer la condamnation. Les plaidoiries et les recours ont pour fonction d'éviter que les peines appliquées ne soient trop sévères.

31 Le Livre des procès et sentences criminelles recense d'une manière presque exhaustive les affaires traitées en grand criminel entre 1755 et 1792.

3245 sont relatives à des écrits ou des libelles séditieux, 118 condamnations sont prononcées contre des contumax. Or, sur 85 affaires concernant des accusés détenus, 78 ont été plaidées par un avocat.

33 PC 9602, l'abréviation PC suivie d'un numéro désigne les procès criminels I ire série conservés aux Archives d'État de Genève. 
Ces sources offrent le matériel nécessaire pour une étude plus fine quant à leur contenu $^{34}$. Ces documents juridiques, dont le volume varie entre 2 et 160 folios, permettent d'identifier un modèle stéréotypé d'argumentation. Bien que le type de délinquance ne soit pas homogène, des récurrences apparaissent dans la manière de conduire la défense. Ces récurrences traduisent des mécanismes standards de défense qui permettent une lecture de la pratique de l'arbitraire par les avocats plaidant devant un tribunal criminel d'Ancien Régime.

\section{L'ARBITRAIRE DÉCONSTRUCTIF}

Arbitraire: ce qui n'est pas défini, ni limité par aucune loi ou constitution expresse, mais qu'on laisse uniquement au jugement et à la discrétion des particuliers $^{35}$.

En France, dès le milieu du siècle, de vives critiques s'élèvent contre l'arbitraire des juges en matière de peines ${ }^{36}$. La justice criminelle, sous les plumes habiles de Beccaria, Servan, Dupaty ou Voltaire, est passée au crible afin de souligner ses imperfections et les scandales qu'elle provoque. Les attaques des Réformateurs du droit pénal ne restent pas cantonnées à l'intérieur du royaume. En effet, des brochuriers genevois portent sur la scène publique les insuffisances ou les abus du système judiciaire de la République ${ }^{37}$. Nées peu après la condamnation de L'Émile et $D u$ contrat social de Rousseau en 1762, les critiques de ces publicistes s'attaquent à la justice criminelle qu'ils décrivent comme un système inique qui condamne d'innocentes victimes. En établissant un lien étroit entre le judiciaire et le gouvernement de la République, les brochuriers sapent le système politique ${ }^{38}$. Malgré cette image «despotique» de la justice criminelle, véhiculée dans ces pamphlets qui inondent la cité genevoise, le cadre légal de la répression paraît garantir une justice modérée. En effet, dès 1738, la question préparatoire disparaît, alors qu'en France elle n'est abolie qu'à partir de 1780 . Depuis 1728 , la roue n'est plus appliquée et les sentences capitales diminuent. En 1734, la défense est légalisée. Entre 1755 et 1791, seules treize sentences de mort sont exécutées alors qu'entre 1700 et 1754 trente-cinq condamnés subissent la peine capitale ${ }^{39}$. Par ailleurs, à Genève, si le Petit Conseil possède un pouvoir arbitraire dans le prononcé d'une peine, il peut être qualifié d'arbitraire mitigé ou limité. En effet, c'est après avoir entendu le Réquisitoire du Procureur général, que les membres du Conseil prononcent la condamnation. Les «Conclusions» de la partie publique proposent aux magistrats une peine qui a été motivée selon diverses sources. D'une part, les références au droit romain, aux jurisconsultes ou à la jurisprudence genevoise et étrangère constituent une base légale éparse qui motive la peine requise. D'autre part, les circonstances du délit consti-

34 Pour la période 1734-1792, j'ai répertorié plus de 80 requêtes (recours en grâce et plaidoiries confondus).

35 Encyclopédie Diderot et d'Alembert, T.1, art. «Arbitraire», 1751.

36 Pour de nombreuses références bibliographiques voir Royer (1995, pp. 157-228).

37 Porret (1992).

38 Porret (1992, p. 145).

39 Porret (1995, p. 390 et p. 48). 
tuent un deuxième élément qui entre dans le choix de la peine. Ainsi, l'arbitraire du Petit Conseil en matière de peine est mitigé, puisque les condamnations prononcées suivent 9 fois sur 10 les «Conclusions » du Procureur général.

Ainsi, s'oppose à l'image négative de l'arbitraire, véhiculée par les pamphlets, l'humanisation de la procédure qui par des modifications du cadre légal (avocat, disparition de la torture, etc.) propose un arbitraire positif de la justice d'Ancien Régime. De plus, au Procureur général qui ancre les sentences du Petit Conseil dans un proto-légalisme et encadre la répression par un arbitraire constructif, les avocats répondent en élaborant un raisonnement analogue qui utilise un arbitraire déconstructif, pour défendre le point de vue opposé à la partie publique. Car, comme les Procureurs généraux, les avocats opèrent selon le mode de pensée: preuve - qualification - motivation. L'argumentation des avocats s'oppose à celle développée par la partie publique, puisque par une méthode déconstructive, les défenseurs critiquent la validité des preuves, pour ensuite proposer des indices ou présomptions d'innocence: ils déqualifient le délit. Sur cette base, ils requalifient le crime: ils opèrent souvent selon un changement terminologique et une réévaluation de la «responsabilité » du prévenu. Enfin, sur cette nouvelle qualification, ils motivent, selon le droit romain, la jurisprudence ou les idées des réformateurs, leur demande de clémence.

\section{DÉQUALIFIER LE CRIME}

Les avocats utilisent une technique déqualificatoire afin de réfuter les preuves du délit et constituer des présomptions d'innocence. Ils prescrivent la légalité comme fondement de l'accusation en exigeant des magistrats le respect des règles légales, lorsque ces derniers cherchent à prouver la culpabilité du prévenu. Puis, les avocats s'orientent vers le principe d'une intime conviction pour fournir une explication de l'acte délictueux. Ils vont donc opérer un glissement: d'une certitude de la culpabilité fondée sur des preuves légales, ils vont ensuite élaborer des présomptions d'innocence en faveur du prévenu.

Les défenseurs s' attardent surtout sur ce qui constitue, selon les criminalistes «la reine des preuves »: le témoignage ${ }^{40}$. Les règles liées à la déposition des témoins sont légales, puisque prescrites par les Édits. Alors que l'accusé n'est pas assermenté au moment de l'interrogatoire, afin d'éviter qu'il ne soit parjure, les témoins doivent avoir prêté serment lors de leur déposition et du récolement ${ }^{41}$. Ces règles doivent être respectées afin de fournir aux magistrats la certitude légale de la culpabilité du prévenu. Si elles sont transgressées, cela permet aux avocats de réfuter la validité des témoignages. Lors d'un recours à la grâce au Conseil des CC, un avocat requiert que la déclaration de l'un des complices de l'accusé soit mise hors du sac.

40 L'aveu de l'accusé constitue également une preuve recherchée par l'Auditeur de Genève (juge instructeur). La pratique pénale met en évidence que, même si le prévenu a avoué, son aveu est souvent ambigu ou incomplet. En effet, le voleur peut avouer le vol d'un drap et réfuter en avoir volé plusieurs, le receleur peut avouer avoir vendu des objets, mais refuser de reconnaître qu'il connaissait leur provenance illicite, l'homicide peut avouer son geste meurtrier, mais réfuter une volonté de donner la mort, etc. Si les officiers de justice cherchent à obtenir l'aveu d'un accusé, tous ces exemples montrent que c'est insuffisant et que les déclarations des témoins sont nécessaires pour obtenir la certitude légale du délit commis.

41 Édits civils de 1714, art. XII du Titre XII, Des causes et des matières criminelles. 
Puisque ce complice a déjà été jugé et condamné, il n'a donc pas été assermenté lors de sa déposition. Les cas d'infamie par jugement contradictoire interdisent de prêter serment, c'est notamment ce point que soulève l'avocat Janot en 1782: «Et d'ailleurs, il faut ne pas perdre de vue que [le complice] a fait sa déclaration après avoir été flétri par une sentence qui l'a condamné au fouet public, la hart au col, et aux galères pour vingt ans, que la religion du serment n'est point intervenue dans cet acte, enfin qu'il est son accusateur [...], et qu'ainsi sa déclaration ne prouverait rien juridiquement ${ }^{42}$. Cette obligation légale de prêter serment lors des témoignages offre ici à l'avocat la possibilité de rejeter une déposition à charge. Il exige donc que le fondement de l'accusation respecte les règles légales.

En plus de cette légalité à laquelle ne doit déroger aucune pièce de la procédure, les avocats prescrivent aux magistrats de se conformer à une règle juridique issue du droit romain: «la théorie des témoins nécessaires». Dès le Moyen Âge, l'arbitraire du juge en matière de preuve est limité par la théorie des «témoins nécessaires » ${ }^{43}$. Cette théorie stipule que deux témoins oculaires irréprochables sont indispensables pour condamner un accusé. En l'absence, aucune peine ne peut être infligée à l'accusé. Cette exigence permet de constituer une preuve complète du crime. La recevabilité des témoignages est fonction du sexe, de l'âge, de la qualité des témoins, des liens de parenté, etc. Par exemple, les enfants de moins de 14 ans, les infâmes, les pauvres, les associés, les complices, les domestiques ne peuvent témoigner contre un accusé. Dans les affaires graves, cette exigence, selon les doctrinaires du droit, doit être appliquée avec rigueur. Le fondement légal de la condamnation est donc issu de cette théorie des témoins nécessaires. De là, naît un système de calcul, selon lequel un témoin oculaire irréprochable constitue une demi-preuve. Les juges en viennent donc à comptabiliser les preuves pour parvenir à une preuve pleine et complète sans laquelle, il n'est pas possible de condamner. En effet, en l'absence de cette preuve complète, il est préférable de laisser échapper un coupable que de condamner un innocent. Cette rigueur procédurale va connaître un assouplissement durant les XIII ${ }^{\mathrm{e}}$ et $\mathrm{XIV}^{\mathrm{e}}$ siècles. Avec l'apparition de la procédure inquisitoire, l'arbitraire du juge en matière de preuve est croissant. Au XVIII ${ }^{\mathrm{e}}$ siècle, l'arbitraire des juges en matière de preuve est une pratique très répandue dans tout le royaume français. Il devient alors possible, dans des cas de crime atroce et dont la preuve est difficile à établir d'entendre des témoins «inhabiles». Les dépositions des témoins sont alors laissées à l'entière appréciation des juges. Comme le précise notamment Daniel Jousse, en 1771 , lorsqu'il s'agit de témoins reprochables, tels que les mendiants, les vagabonds: «[...] il dépend entièrement de la prudence du juge, d'arbitrer quel égard on doit avoir à la déposition de ces sortes de personnes ${ }^{44}$. Ainsi, la maxime «les preuves plus claires que le jour» n'est plus appliquée. Et il faut condamner, même à la mort, dans les cas de crime grave, plutôt que de laisser échapper un éventuel coupable.

Les avocats genevois, quant à eux, exigent que l'accusation respecte la théorie des témoins nécessaires, puisque a priori elle favorise la protection des innocents. Si l'incrimination ne repose pas sur la déposition de deux témoins oculaires irréprochables, alors le doute doit bénéficier à l'accusé. Les magistrats ne peuvent pas se

\footnotetext{
42 PC 13764,1782 , recel, recours fol. $509 \mathrm{v}^{\circ}$.

43 Schnapper (1965).

44 Jousse (1771, V. 1, p. 700).
} 
baser sur des indices qui formeraient des présomptions pour condamner un prévenu. Cette exigence est réitérée pratiquement dans toutes les requêtes, comme le réclame notamment l'avocat Calandrini dans un recours de 1784: «Pour condamner justement il faut que le juge ait acquis la preuve complète du crime. Pour que cette preuve soit complète, il faut comme le dit le célèbre Risi dans ses Observations sur la jurisprudence criminelle, que le corps du délit soit bien vérifié selon le prescrit des lois; que, deux témoins non suspects et assermentés, déposent avoir vu commettre l'action, que ces témoins soient parfaitement d'accord entre eux, et assurent le tout en face du [prévenu], qu'alors il en résulte une preuve pleine, complète et légale, mais que ce n'est qu'à la vue d'une telle preuve que les juges seront fondés à prononcer leur sentence ${ }^{45}$. Aucune incertitude n'est permise et les juges doivent avoir la certitude légale du crime. En cas de doute, l'innocence doit prévaloir. Ainsi, en opposition avec la pratique du Procureur général et des magistrats qui acceptent des témoins reprochables et condamnent en cas de fortes présomptions sur soupçons véhéments, certains avocats rejettent totalement ce principe arbitraire en matière de preuves, lorsque celui-ci sert à prouver la culpabilité du prévenu.

Si les avocats exigent un fondement légal pour la preuve des délits, ils développent le principe de l'intime conviction, quand il s'agit de prouver l'innocence du prévenu ou sa culpabilité atténuée. Leur démarche est sélective: la preuve légale pour l'accusation et l'intime conviction pour la défense. Dans son recours à la grâce en faveur d'un voleur de blé, en 1779, l'avocat Rigaud insiste sur la nécessité de condamner sur une preuve légale, car les effets $«[\ldots]$ de l'arbitraire qui règne nécessairement dans le calcul de la preuve conjecturale [...] $\rangle^{46}$ sont dangereux. Les faits contenus dans le procès ont été examinés minutieusement et rejetés; la procédure a été critiquée du fait de son manque de formalité et les témoignages sont suspectés de faux. Cette démarche mène l'avocat à détruire tous les éléments qui constituaient une certitude légale pour les juges. Finalement, l'avocat introduit des indices qui tendent à prouver l'innocence du prévenu: «[...] le suppliant a pour lui des présomptions directes, qu'il va mettre très humblement sous les yeux de vos Excellences en leur demandant respectueusement encore quelques instants d'attention ${ }^{47}$. Si l'accusé était coupable il aurait eu un comportement différent de celui qui a été établi par l'enquête. En effet, l'avocat fonde son raisonnement sur la maxime suivante: toute personne coupable d'acte illicite agit de façon à ne pas éveiller les soupçons et donc tous ses actes tendent à masquer son délit. Par exemple, le voleur n'affichera pas les objets dérobés, il cherchera à les revendre discrètement, etc. Les présomptions d'innocence en faveur de l'accusé postulent donc de celui-ci un comportement rationnel qui vise à étouffer les soupçons. Or, le prévenu ne s'est pas conformé à cette règle de discrétion, car «[...] ici, il y avait pour ainsi dire publicité, car l'accusé avait-il un ou deux complices attitrés pour porter son blé? Non, il employait le premier venu. Où les venait-il chercher? Sur le port, à quelle heure ! En plein jour. [...]. Leur demandait-il le secret. Nullement [...] $\gg^{48}$. Cette argumentation fonde des indices de la non-culpabilité de l'accusé. Si le prévenu avait commis le crime dont on l'accuse, alors son comportement aurait été différent: il aurait agi

\footnotetext{
45 PC 14236bis 1784 , vols, recours fol. 521-521 vo, souligné dans le texte.

46 PC 13252 (bis et ter), recours fol. 705.

47 PC 13252 (bis et ter), recours fol. 735.

48 PC 13252 (bis et ter), recours fol. 738 .
} 
dans le secret, de façon à cacher son délit. La publicité des actes délictueux constitue dans l'argumentation des avocats genevois des indices qui, très systématiquement, sont utilisés par les défenseurs pour n'importe quel type de délit et qui servent à consolider les présomptions d'innocence.

Les exigences pour l'accusation sont très rigides et la condamnation ne doit reposer que sur des preuves légales et sur la théorie des témoins nécessaires. Puis, l'avocat met en évidence les présomptions en faveur de l'accusé qui visent à introduire le doute de la culpabilité dans l'esprit des juges. Ainsi, la théorie des témoins nécessaires comme fondement de la preuve légale est exigée, alors que l'intime zonviction sert à la décharge de l'accusé. Les avocats utilisent la légalité selon un processus «négatif » et la certitude morale selon un processus «positif». La légalité sert à déconstruire l'accusation et l'intime conviction à fonder la défense.

Alors que le droit savant avait élaboré la théorie des témoins nécessaires pour mieux préserver l'innocence de l'accusé, on observe que cette théorie est utilisée par les avocats pour nier le fondement légal de l'accusation; en outre, elle est contournée pour établir une conviction d'innocence de l'accusé. L'obligation de respecter des règles légales faite aux magistrats sert à rejeter l'accusation. Les avocats déqualifient les délits, puisqu'ils déconstruisent le système de preuves servant à fonder le chef d'accusation. Ensuite, ils élaborent l'innocence ou la responsabilité atténuée du détenu sur des présomptions ou des indices. Il y a un glissement de la légalité vers l'arbitraire. Sur cette base de preuve réévaluée, ils cherchent à «requalifier» le délit.

\section{REQUALIFIER LE CRIME}

Le degré plus ou moins grand de malice, les motifs qui ont porté au crime, la manière dont il a été commis, les instruments dont on s'est servi, le caractère du coupable, la récidive, l'âge, le sexe, le temps, les lieux, etc. contribuent pareillement à caractériser l'énormité plus ou moins grande du crime; en un mot l'on comprend sans peine que le différent concours des circonstances qui intéressent plus ou moins la sûreté des citoyens, augmente ou diminue l'atrocité des crimes $^{49}$.

L'acte qualificatoire qui appartient à Genève au Procureur général, est décisif, car il induit la peine requise contre le prévenu. La partie publique établit, dans son Réquisitoire, une description minutieuse des «circonstances» du délit qui sont de deux ordres: matérielles et morales. D'une part, les circonstances extérieures résultent de la manière dont le crime a été commis. En effet, la partie publique détermine si le délit a été commis de jour ou de nuit, avec ou sans effraction, avec ou sans fausse clé, etc. D'autre part, utiles pour estimer la dangerosité du criminel, les circonstances biographiques, telles que les mœurs, la récidive etc., sont considérées pour qualifier le délit. La qualification sert à la fois à nommer le délit, par exemple à distinguer un assassinat d'un meurtre sans «intention de donner la mort », et à

49 Encyclopédie Diderot et d'Alembert, art. «Crime » signé Boucher d'Argis, T. 4, 1754. 
évaluer le degré de dangerosité du prévenu. Bien qu'établie par la doctrine $\mathrm{e}^{50}$, la qualification reste arbitraire, car le Procureur général la fonde sur des évaluations morales qu'il choisit de retenir afin de mesurer la dangerosité du criminel. Les avocats, se calquant sur la partie publique n'effectuent pas la qualification des délits, mais leur déqualification. Ils jouent sur deux registres. Premièrement, si l'affaire s'y prête, ils vont opérer un changement terminologique. Deuxièmement, les avocats cherchent à réévaluer l'intention criminelle, non pas selon des éléments biographiques, mais en invoquant des circonstances sociales (pauvreté, parenté, etc.) qui expliquent, voire induisent le crime; ils élaborent un déterminisme social criminogène.

Les cas d'homicides, pour lesquels les avocats présentent une plaidoirie ou un recours à la grâce, sont emblématiques du changement terminologique opéré. En effet, ces affaires d'homicide possèdent presque toutes les mêmes caractéristiques. Selon les avocats, les accusés n'ont pas prémédité leur meurtre. Les prévenus n'ont agi que par simple réflexe défensif; ils se sont laissés emporter par la colère ou l'ivresse; ils ont répondu à une menace ou une injure. Toutes les dépositions à charge sont minutieusement critiquées par les avocats qui les écartent, car tel témoin est soi-disant créancier de l'accusé, tel autre était trop loin au moment des faits, il n'a pas vu correctement ce qui s'est réellement passé, etc. Ainsi, les avocats retiennent uniquement quelques témoignages en faveur des accusés, qui proviennent parfois de membres de la famille ou d'amis. La principale source d'information sur le crime est tirée essentiellement des aveux de l'accusé qui désigne, par exemple, son délit comme un mouvement de colère. La victime a, peu avant sa mort, provoqué le prévenu et comme «la nature a imprimé dans le cœur de tous les hommes le juste désir de se défendre contre la violence et de repousser les injures $»^{51}$, c'est à son corps défendant que le prévenu a tué. Car, tout concourt à montrer que «[...] le suppliant ne l'a point tué à la suite d'un dessein prémédité $»^{52}$. Ainsi, c'est en réponse à une provocation, une injure, voire même une menace que le prévenu a eu un geste homicide. La non-préméditation, souvent associée au hasard d'une rencontre, l'emportement de la colère et parfois l'ivresse, sont toutes des circonstances qui tendent à prouver, selon les avocats, que dans huit cas sur dix les prévenus ne sont pas coupables d'homicide, mais qu'ils ont agi en état de légitime défense: «Ce fait enfin [la menace] met le suppliant dans le cas de la légitime défense au moment même où il a tué T.» ${ }^{53}$. Ainsi, les avocats opèrent un déplacement dans la qualification du délit, de l'assassinat, ils évoquent la légitime défense qui atténue l'intentionnalité coupable du prévenu. En effet, se défendre contre une attaque, une menace ou une injure n'implique pas la même intention de dol envers la victime et la condamnation doit donc être moins sévère. L'homicide est donc requalifié comme étant de la légitime défense.

Une deuxième technique est utilisée, afin de parfaire la réévaluation de la dangerosité du criminel. Les avocats dressent le portrait d'un criminel qui n'est pas "scélérat dans l'âme» - ils refusent l'image d'un accusé pécheur - pour ancrer le

\footnotetext{
so Muyart de Vouglans (1781, Titre IV du Livre I, Vol. 1).

51 PC 9096, 1744, plaidoirie fol. 186 $\mathrm{v}^{\circ}$.

52 PC 12900,1777 , recours fol. $330 v^{\circ}$.

53 PC 12900, recours de l'avocat Rigaud, fol. 342.
} 
délinquant dans une spirale sociale et familiale qui l'entraîne sur le chemin du crime. Bien que déjà fragilisés, les accusés ne se sont pas engagés immédiatement dans la délinquance, mais leurs tentatives d'insertion sociale se sont soldées par un échec. En effet, souvent confrontés à leur fragile constitution, les prévenus n'ont pas de travail stable. Ainsi, l'emploi faiblement rémunéré qu'ils occupent, devient très précaire lors de leurs nombreuses maladies et le seuil de subsistance n'est plus assuré. Cherchant à sensibiliser les magistrats, les avocats évoquent la circonstance atténuante du crime par besoin afin d'émouvoir les juges: «Son salaire, diminué par sa faiblesse, suspendu pendant ses maladies durant l'hiver, a bientôt cessé de lui fournir les choses les plus nécessaires et l'a laissé presque nu sur la terre, mourant de faim et engourdi par le froid $»^{54}$. Expliquant les vols ou le libertinage par la précarité sociale et la misère, les avocats diminuent la gravité de ces délits en invoquant des besoins alimentaires non assouvis. Parfois, à cette motivation atténuante s'ajoute la traîtrise de l'entourage du criminel qui a provoqué sa perte. En effet, les accusés tentent aussi d'expliquer leur délit en prétextant qu'un ami ou une connaissance les a poussés à le commettre. La séduction des incitateurs trouve écho chez l'accusé affaibli par la misère, notamment lorsque celui-ci a des charges de famille, car comment répondre "[...] par des leçons de morale à ses petits enfants qui lui demandent du pain?» ${ }^{55}$. L'accumulation des circonstances sociales constitue, dans l'économie argumentative des avocats, des données causales qui servent à montrer la paralysie de la volonté du prévenu. Le libre consentement, ayant souvent pour caractéristique une faible éducation, disparaît face à des contraintes extérieures qui relèvent de besoins physiologiques ou sociaux.

L'apport assez nouveau réside dans l'invocation de l'environnement social ou familial comme déterminisme criminogène. Les avocats jouent sur la «non-responsabilité » ou sur une responsabilité atténuée de l'individu à cause de faits sociaux. Certains défenseurs supposent des éléments de types économiques ou familiaux détruisant la liberté morale de l'accusé. La causalité du délit est alors introduite en étroite relation avec milieu social du prévenu. Les accusés sont ce que la société en a fait. En 1779, un avocat justifie un vol en mettant en évidence que la condition économique du prévenu est une cause de sa culpabilité: «Ne perdez jamais de vue que sans sa pauvreté, il eut toujours été innocent ${ }^{56}$. Le comportement illicite a donc été déterminé par des faits socio-économiques et non pas par une volonté coupable. Le même type d'argument est utilisé en 1784 par l'avocat Calandrini, qui cite Beccaria, pour expliquer un autre vol: «D'ailleurs ce crime est commis ordinairement par des hommes pauvres et par ces malheureux auxquels le droit de propriété (droit terrible et qui n'est peut-être par nécessaire) n'a laissé que la simple existence ${ }^{57}$. Les prévenus sont conditionnés par une logique sociale qui ne leur laisse aucune chance d'échapper à leur sort. Ils sont soumis à des déterminismes socio-économiques qui les conduisent inéluctablement au crime. La pauvreté entraîne des dispositions criminelles.

Les avocats ont donc requalifé le délit pour diminuer sa gravité en le renommant. Par ailleurs, certains construisent des images d'accusés qui ne possèdent qu'une res-

54 PC 15443, 1788, vols, recours de l'avocat Claparède, fol. 103.

ss PC 15983, 1792, maquerellage, recours de l'avocat Claparède, fol. $51 \mathrm{v}^{\circ}$.

56 PC 13263, plaidoirie de l'avocat Cramer fol. $116 \mathrm{v}^{0}$.

57 PC 14236 bis, recours fol. 5. 
ponsabilité limitée ou inexistante de l'acte délictueux. Ils attribuent les causes du délit à des déterminismes sociaux, ce qui leur permet de nier ou d'atténuer l'intention coupable des accusés. Ils construisent un stéréotype de criminel, dont la responsabilité délictueuse doit être imputée à un environnement social caractérisé par la pauvreté. Après ce travail de requalification, les avocats demandent qu'une peine plus adéquate soit motivée sur la base de cette nouvelle qualification.

\section{MOTIVER LA DEMANDE DE CLÉMENCE}

Comment les avocats se positionnent-ils vis-à-vis de l'arbitraire des magistrats en matière de peine? Les études sur les réformateurs du droit ont montré que, dès les années 1760 , la légalité des peines doit se substituer à l'arbitraire des juges. Comme le pouvoir des juges en matière de châtiment est considéré comme despotique et tyrannique, les philosophes et théoriciens du droit exigent la légalité. "Lorsqu'un code formel de lois devant être observées à la lettre ne laisse au juge d'autre tâche que d'examiner les actes des citoyens et de déterminer s'ils sont conformes ou contraires à la loi écrite, lorsque les normes du juste et de l'injuste qui doivent régler les actions de l'ignorant comme du philosophe sont une question non de controverse, mais de fait [... ${ }^{58}$ la tyrannie disparaît. La controverse se déroule dans l'opinion publique. Or, qu'en est-il au niveau de la pratique judiciaire? À Genève au XVIII' ${ }^{e}$ siècle, l'arbitraire des juges n'est pas, pour la défense, un élément qui accentue la sévérité de la répression. Au contraire, les instruments juridiques utiles pour la motivation des peines offrent un large éventail, dans lequel les avocats puisent ce dont ils ont besoin, pour requérir la clémence du glaive.

Bien qu'ils constatent l'absence d'un code pénal, ou code criminel, qui fixe les peines aux délits, peu d'avocats prônent la légalitét ${ }^{59}$. Ils exploitent les avantages de l'arbitraire constructif qui contraint les magistrats à se référer à la jurisprudence. En effet, comme le précise l'avocat Vasserot de Chateauvieux, en 1755, dans sa plaidoirie « [...] la jurisprudence sur les peines doit être uniforme, ce principe est aussi sévère que celui qui statue la punition des crimes $\|^{60}$. Les sentences antérieures ayant force de loi, les membres du Conseil doivent se conformer à celles rendues par le passé. Les références à la jurisprudence ne se limitent pas uniquement aux procès jugés à Genève. En effet, des analogies aux jugements rendus par le Sénat de Savoie ou par les Parlements français, garantissent la peine requise par l'avocat. En l'absence de code qui fixe les peines à chaque type de délit, les procédures criminelles font office d'autorité en matière de peine. Comme le souligne déjà l'avocat P.A. Rigaud en 1772, «[...] puisque nous n'avons pas de lois pénales, Vos Excellences se gouverneront par les arrêts les plus doux rendus en cas pareils ${ }^{61}$. Par ce manque de

58 Beccaria (1764, \& IV).

59 Le seul réclamant dans sa plaidoirie un code pénal, est l'avocat Étienne-Alexandre Bousquet, (PC 15996, 1790, paroles séditieuses). Cet avocat profite d'un procès politique pour dénoncer clairement les aspects de la justice qui sont devenus intolérables à la fin du siècle, comme l'absence d'un code pénal, l'arbitraire des juges, la non-publicité des procédures, etc. Il préconise la suppression pure et simple de l'ordre judiciaire. Sa plaidoirie sera censurée par le Petit Conseil pour propos injurieux contre les témoins.

60 PC 10210, 1755, vol domestique, plaidoirie de l'avocat Vasserot de Châteauvieux, fol. $309 \mathrm{v}^{\circ}$.

61 PC 13263, vol, plaidoirie fol. 118. 
fixité des peines, les magistrats doivent rendre des jugements cléments et surtout ne pas s'éloigner des cas jurisprudentiels.

Or, les cas de jurisprudence ne proposent jamais des délits strictement identiques. En effet, chaque délit étant qualifié en fonction de ses multiples circonstances, un crime est donc unique. Un vol, par exemple est commis de nuit avec ou sans effraction, avec ou sans violence, etc.; il ne peut fournir qu' un nombre restreint de circonstances analogues avec un autre vol. Dans ce vaste choix de la jurisprudence qui s'étend notamment aux sentences rendues par des tribunaux étrangers, les avocats se réfèrent à une grande quantité de délits et de peines pour motiver leurs arguments. Les analogies sont effectuées systématiquement à l'avantage du prévenu défendu. L'utilisation des cas jurisprudentiels est donc arbitraire, il sert toutefois à réclamer des sentences modérées en faveur des prévenus. Une procédure criminelle est intentée en 1784 contre un voleur. Lors du recours en grâce qui vise à éviter au prévenu la pendaison, l'avocat Mallet-Butini se réfère à une affaire similaire, datant de 1773. Il utilise l'exemple d'un procès, intenté onze ans auparavant contre un voleur, afin de déqualifier le crime pour lequel il plaide. Le défenseur compare le degré de gravité entre un vol avec effraction et un vol à main armée. La technique, développée par Mallet-Butini, compare deux situations, pour faire pencher la balance en faveur du prévenu qu'il défend. Cette comparaison veut prouver que le crime du prévenu recourant est moins qualifié que celui qui a été commis onze ans auparavant; la peine doit donc être moins sévère ${ }^{62}$.

En l'absence de fixité des délits et des peines, la jurisprudence acquiert force de loi. Les magistrats sont donc limités dans leur pouvoir discrétionnaire en matière de peine. Malgré les critiques que l'on trouve, notamment sous la plume de Boucher d'Argis qui souhaiterait des lois immuables et qui déplore «[...] l'incertitude de la jurisprudence sur la plupart des questions, soit par la contradiction apparente ou effective des lois, soit par la diversité d'opinions des auteurs, ou par la diversité qui se trouve entre les jugements des différents tribunaux, et souvent entre les jugements d'un même tribunal "63, les jugements rendus ne sont pas contradictoires entre eux. L'étude de la pratique pénale à Genève, a montré comment les «Conclusions » des Procureurs généraux (neuf fois sur dix suivies par le Petit Conseil) s'orientent sur le chemin de la légalité, et comment, notamment, elles s'ancrent dans la jurisprudence genevoise ou étrangère ${ }^{64}$. Les avocats qui demandent aux magistrats de suivre la règle formée par les jugements antérieurs s'inscrivent parfaitement dans ce courant de pensée et de pratique. Ils font valoir la dangerosité des crimes précédents, dans le but de souligner la moindre gravité du délit pour lequel ils plaident. Ils opèrent donc avec un arbitraire «limité » et sélectionnent les cas de jurisprudence les plus pertinents et avantageux pour les prévenus qu'ils défendent.

L'arbitraire dans le prononcé des peines permet aux avocats d'être détachés d'un code fixe qui limiterait leurs choix de comparaison. L'arbitraire en matière de peine, par sa définition «positive» (non fixée par la loi) et par sa pratique, permet donc des

62 PC 14236.

${ }^{63}$ Encyclopédie méthodique, Jurisprudence, art. «Jurisprudence», Vol. 5. On peut lire dans cet article: «Encore si les lois de chaque pays étaient fixes et immuables, la jurisprudence ne serait pas si immense qu'elle est ; mais il n'y a presque point de nation, point de province, dont les lois et les coutumes n'aient éprouvé plusieurs variations [...]».

64

Porret (1995). 
stratégies de défense mouvante qui sont ignorées, semble-t-il, par les Réformateurs du droit, lorsqu'ils réclament un code pénal.

Dès 1750 environ, pour motiver leur demande de clémence, les défenseurs cherchent dans la philosophie pénale des Réformateurs du droit les instruments théoriques qui sont susceptibles d'inciter les juges à la clémence. En effet, les idées de modération et les thèses utilitaires de Montesquieu ou de Beccaria, tiennent lieu d'arguments usuels pour la défense. Parfois favorables à la prévention générale, les avocats soulignent qu'il est important «[...] que le coupable reçoive une peine proportionnée à son délit; que cette punition soit publique, il faut que l'appareil du châtiment en impose au peuple et qu'un exemple effrayant détourne du chemin du crime ceux qui seraient tentés d'y entrer. Il faut que cette peine fasse impression sur le coupable et qu'elle soit propre à le corriger. Voilà tout ce qu'exige la sûreté publique ${ }^{65}$. Les avocats genevois sont ainsi ouverts à la culture juridique des Lumières qu'ils imprègnent dans la pratique pénale de la République. Ils ne proposent pas une réflexion sur le droit de punir, mais plutôt sur la fonction que remplit la peine. Ils en discutent la finalité exemplaire, utilitaire et corrective.

À Genève durant le XVIII ${ }^{e}$ siècle, les sentences sont lues publiquement, parfois à son de trompe devant l'Hôtel de Ville ou aux carrefours de la ville. Les exécutions capitales, les châtiments corporels et infamants, les exécutions en effigie pour les criminels contumaces, sont effectués la plupart du temps sur la place publique. Cette symbolique de l'appareil répressif met en jeu la représentation du pouvoir politique et judiciaire et répond entre autres à un objectif: dissuader les criminels potentiels par le spectacle du châtiment et inspirer l'horreur du crime aux populations. Cette «pédagogie de l'effroi ${ }^{66}$ se fonde sur l'exemplarité de la peine infligée. Dans les années 1760 , les Réformateurs s'orientent vers une finalité de la peine différente. En effet, substituant l'utilité à l'exemplarité, la peine doit davantage viser à corriger le criminel, afin de le rendre meilleur, qu'à édifier les populations. L'accent est alors mis sur une éducation morale et une édification interne qui vise au respect des lois de la société et qui ouvre sur une réelle utilité sociale.

Volontiers utilitaristes, les avocats genevois adhèrent à ces nouvelles conceptions punitives, lorsqu'ils motivent la peine qu'ils réclament pour les prévenus. Ils mitigent l'effet que peut procurer une exécution capitale sur le criminel potentiel, car «[...] le scélérat qui entreprend le métier de brigand se joue de sa vie et la perd sans regret $[\ldots]{ }^{67}$. De plus, le cas du complice, ayant déjà été jugé et puni, fournit un exemple suffisant pour la société. Le peuple est davantage impressionné par la répétition d'une punition clémente que par la ponctualité d'une sentence sévère. Comme l'écrit Beccaria en 1764, c'est la fréquence qui a une valeur pédagogique, plutôt que la vue d'un châtiment atroce et rare: «Or, lorsque quelques crimes se multiplient par des circonstances particulières, le nombre des châtiments répétés, quoique dictés par la clémence, font plus d'impression sur le peuple, que des punitions très sévères, mais très rares, et dont le souvenir s'efface bientôt ${ }^{68}{ }^{6}$. Ainsi, l'exemplarité d'une sentence douce est plus utile, puisqu'elle marque plus durablement les consciences.

\footnotetext{
65 PC 11810, 1769, vol, recours des avocats Des Arts et Dunant, fol. 258.

66 Porret (1994).

67 PC 14236, 1784, vols, recours de l'avocat Mallet Butini, fol. 5-5 $\mathrm{v}^{\circ}$.

68

PC 12260, 1772, vols, recours de l'avocat Prévost, fol. $114 \mathrm{v}^{\circ}$.
} 
En plus de cette fonction pédagogique, le châtiment infligé s'oriente vers une finalité corrective. La punition doit impressionner le peuple, pour le détourner de commettre des crimes semblables, mais surtout elle doit corriger le coupable. Comme le rappelle l'avocat Prévost en 1772, le magistrat doit châtier pour corriger, «[...] punir et non pas flétrir, s'il est possible, afin de laisser au criminel assez d'énergie pour élever son âme abattue par tant de circonstances malheureuses, en lui laissant l'espérance touchante et lumineuse de pouvoir un jour n'être plus avili, mais vertueux ${ }^{69}$. Or, le délinquant "régénère son cœur corrompu » par le travail. C'est ainsi que l'enfermement couplé au labeur possède deux avantages: celui d'être utile à la société et celui de corriger le coupable. Le criminel «[...] a fait tort à la société par ses crimes, c'est donc en le rendant utile à cette même société par son travail qu'il faut réparer ses forfaits, c'est en le montrant le reste de ses jours, couvert de fers, et courbé sous le faix du travail que vous ferez trembler le fainéant, qui est déjà scélérat dans son cœur et non pas un châtiment regardé par lui comme la fin de tout, châtiment qu'il voit le matin et qu'il oublie le soir ${ }^{70}$. Ici, l'avocat Mallet-Butini inspiré par Beccaria, alors qu'il présente une requête en faveur d'un criminel accusé de vols nocturnes en 1784, réfute l'utilité de la peine de mort et propose, dans la suite de son recours, de faire travailler les délinquants dans la cour de la maison de discipline $^{71}$.

\section{CONCLUSION}

Le système de défense utilisé par les avocats lors des procès criminels, est, comme on l'a vu, déconstructif. Les juristes déqualifient le délit, en s'attaquant aux éléments qui fondent l'accusation. Ils trient ou rejettent les preuves, selon des critères tirés de jurisconsultes ou de règles légales. Puis ils fondent des présomptions d'innocence sur la base d'indices. Les défenseurs opèrent une relecture systématique de toutes les pièces à charge, afin d'amener les magistrats à prendre en considération uniquement les données favorables au prévenu. Après ce travail de déconstruction, ils renomment le délit. C'est-à-dire qu'ils démontrent, par exemple, que le criminel n'est pas un meurtrier, mais qu'il a réagi en état de légitime défense à une provocation ou à une menace. Ainsi, le délit doit être qualifié d'homicide involontaire et non pas d'assassinat. C'est en opérant un changement terminologique qu'ils parviennent à requalifier le crime. Selon une autre démarche qui reconsidère l'intentionnalité du criminel, les avocats démontrent en tirant parti d'éléments biographiques, familiaux ou économiques que le prévenu n'est pas entièrement responsable de son acte. Ils jouent, ici encore, sur une réévaluation de l'intention du coupable. Ensuite, ils proposent une nouvelle peine plus adéquate au regard de cette requalification. Les défenseurs cherchent par ailleurs à imposer aux magistrats des contraintes légales jurisprudentielles ou philosophiques pour les convaincre de prononcer des sentences clémentes, voire de commuer une peine. Les condamnations sont laissées à l'appréciation des magistrats, mais elles doivent être justement proportionnées au délit, strictement nécessaires et conformes aux sentences antérieures. $\mathrm{Si}$ les théoriciens du droit au $\mathrm{XVIII}^{\mathrm{e}}$ siècle réclament la légalité des peines,

\footnotetext{
69 PC 12260, vol, recours fol. $116 \mathrm{v}^{\circ}$.

70 PC 14236, 1784, vols, recours fol. $5 \mathrm{v}^{\circ}$.

71 PC 14236.
} 
l'exemple de l'utilisation de la légalité par les avocats montre, notamment en matière de preuve, que cela constitue un cadre très rigide qui n'est utile que pour récuser le fondement de l'accusation. Quand ils examinent les causes du délit, ils dépassent ce cadre rigide pour se placer dans une certitude morale. Quant à l'arbitraire des juges en matière de peine, elle laisse aux défenseurs le choix d'effectuer des analogies de délits ou de peines à l'avantage du prévenu.

Les avocats genevois exploitent donc un espace entre arbitraire et légalité, espace qui peut être qualifié d'arbitraire juridiquement motivé et qui leur permet de développer avantageusement leurs arguments de défense. À ces exigences de légalité développées par les Réformateurs du droit dans l'opinion publique, les avocats dans la pratique judiciaire répondent par une utilisation de l'arbitraire en matière de preuve, de qualification du délit et de la peine.

Après ce rapide rappel de ce que l'on peut appeler le mécanisme standard d'une requête, une question s'impose. Quel est l'impact des requêtes auprès des magistrats? Les membres du Petit Conseil prononcent-ils une peine plus légère, si un avocat a pris la parole pour défendre un prévenu? Le Conseil des CC accepte-t-il de commuer la condamnation ${ }^{72}$ ? Les mécanismes de déqualification, requalification et motivation de la demande de clémence structurant les requêtes des avocats aboutissent, dans $47 \%$ des cas, soit près d'un cas sur deux, à modérer la condamnation ${ }^{73}$. Les défenseurs jouent un rôle majeur dans les affaires criminelles, puisqu'ils ajoutent à la qualification proto-légale du Procureur général, une argumentation juridique qui en modère les conséquences pénales. De plus, les sentences rendues par les magistrats peuvent, elles aussi, être mitigées lors des recours devant le Conseil des CC. En amont de l'arbitraire du Petit Conseil en matière de peine, la partie publique fait déjà office de frein. Le cadre légal ajoute en 1734 un deuxième élément de modération; c'est la défense. En aval, le recours en grâce constitue une seconde limite au pouvoir des magistrats. Les commutations de peines décidées par le CC sont sans appel et elles peuvent, selon les conditions déterminées par la loi, être réitérées sur demande des accusés. Puisque les interventions des avocats sont efficaces environ une fois sur deux, alors elles sont un frein qui endigue l'arbitraire du Petit Conseil en matière pénale.

Si l'on opère une mesure de l'intentionnalité punitive du pouvoir judiciaire avec la pratique pénale, les archives mettent en évidence que ce n'est pas par souci d'élimination systématique des déviants, mais davantage en fonction d'un souci croissant pour les individus et pour la protection de leurs droits que les praticiens rendent

72 Pour pouvoir évaluer la portée de ces deux types de sources, il convient d'adopter une démarche comparative. Dans le cas du recours à la grâce, la lecture est immédiate: il suffit de constater si ce dernier a eu un impact sur les décisions des membres du CC; ils acceptent ou refusent de commuer la condamnation prononcée contre l'accusé. Pour la plaidoirie, comment évaluer la répercussion potentielle, sachant que la sentence n'a pas été prononcée? Si les jugements du Petit Conseil entérinent 9 fois sur 10 les «Conclusions», il est possible de comparer la peine requise par le Procureur général et celle rendue. Dans le cas où cette dernière est moins sévère que celle de la partie publique, on peut admettre que l'avocat ayant plaidé la cause a modéré la peine. Les requêtes peuvent donc être abordées dans une étude quantitative qui permet de mesurer leur efficacité.

73 La base de calcul comprend 86 requêtes dont 19 plaidoiries et 67 recours en grâce. Sur les 19 défenses présentées par les avocats lors des procès criminels, 8 d'entre elles aboutissent à un adoucissement de la peine requise par le Procureur général (soit $42 \%$ ). Sur 67 recours, 32 ont eu pour conséquence une commutation de la condamnation (soit $48 \%$ ). 
la justice d'Ancien Régime à Genève. Les accusés sont des individus qui possèdent le droit de déléguer leur défense et leur recours à des professionnels qui font preuve d'efficacité auprès des magistrats dans près de $50 \%$ des cas. Au fil du siècle, légalisés, les droits des individus à la défense et au recours se détachent d'une condition juridique, notamment, car la défense dépend du libre choix de l'accusé et le recours concerne davantage de prévenus de basse condition. Ainsi, un nombre croissant d'accusés bénéficient d'une modération de peine. Les avocats genevois au siècle des Lumières contribuent à l'émergence d'une pratique judiciaire impérative à la genèse de l'État de droit: ils incarnent une justice criminelle dont la modération est propre aux institutions de la République.

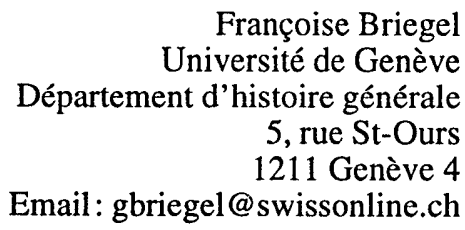

\section{Sources}

Beccaria, C., Des délits et des peines, Paris, 1764.

Bérenger, J.-P., Histoire des derniers temps de la République de Genève, Genève, 1801. Édits civils de la République de Genève, Genève, 1714.

Édit du 11 mars 1768, Genève, 1768.

Encyclopédie, ou Dictionnaire raisonné des sciences, des arts et des métiers, par une société des gens de lettres. Mis en ordre et publié par M. Diderot, de l'Académie Royale des Sciences et des Belles-Lettres de Prusse; et quant à la Partie Mathématiques, par M. d'Alembert, de l'Académie Royale des Sciences de Paris, de celle de Prusse, et de la Société Royale de Londres, (17 volumes de texte et 11 volumes de planches), 1751-1772.

Encyclopédie méthodique, ou par ordre de matières; par une société de gens de lettres, de savants et d'artistes; précédée d'un vocabulaire universel, servant de Table pour tout l'Ouvrage, ornée des portraits de MM. Diderot et d'Alembert, premiers Éditeurs de l'Encyclopédie. Jurisprudence, dédiée et présentée à Monsieur Hue de Miromesnil, garde des Sceaux de France, etc. (10 volumes), Paris, 1782-1790.

Extrait des Registres du Conseil du 14 Juillet 1734, Genève, 1734.

Jousse, D., Traité de la justice criminelle de France, où l'on examine tout ce qui concerne les Crimes et les Peines en général et en particulier; les juges établis pour décider les affaires criminelles; les parties publiques et privées; les accusés; les ministres de la Justice Criminelle; les experts; les témoins; et les autres personnes nécessaires pour l'instruction des procès criminels; et aussi tout ce qui regarde la manière de procéder dans la poursuite des Crimes, Paris, 4 V., 1771.

Livre des procès et sentences criminelles, 2 registres, 1755-1791.

Montesquieu, De l'esprit des lois, Genève, 1748.

Muyart de Vouglans, P.-F., Les lois criminelles de France dans leur ordre naturel, Neuchâtel, 2 V. 1781.

Ordonnance criminelle, Saint-Germain-en-Laye, août 1670, François Isambert, Recueil général des anciennes lois françaises depuis l'an 420 jusqu'à la Révolution de 1789 (30 volumes), Paris, 1821-1833, Tome XVIII, pp. 372-423. 
Règlement de l'Illustre Médiation pour la pacification des troubles de la République de Genève, Genève, 1738.

Papiers Ami de Rochemont, procédures civile et criminelle II, 1739-1792, Mss Hist. 110. Sartoris, J.-P., Éléments de la procédure criminelle suivant les Ordonnances de France, les Constitutions de Savoye, et les Édits de Genève, 2 V., Amsterdam, 1773.

\section{Références}

Astaing, A., Droits et garanties de l'accusé dans le procès criminel d'Ancien Régime (XVI XVIII ${ }^{e}$ siècles). Audace et pusillanimité de la doctrine pénale française, Aix-enProvence, Presses universitaires d'Aix-Marseille, 1999.

Beattie, J.M., Crime and the Courts in England, 1600-1800, Oxford, Clarendon Press, 1986.

Carbasse, J.-M., Introduction historique au droit pénal, Paris, Presses universitaires de France, 1990.

Dupont-Bouchat, M.-S., Pour une meilleure justice. La professionnalisation des procureurs et avocats, L'assistance dans la résolution des conflits. L'Europe médiévale et moderne, Recueils de la Société Jean Bodin, Vol. LXIV, Bruxelles, De Boeck Université, 1997, pp. 229-248.

Perrenoud, A., La population de Genève du seizième au début du dix-neuvième siècle. Étude démographique, Vol. 1: Structures et mouvements, Genève, Mémoires et documents publiés par la Société d'histoire et d'archéologie de Genève, Tome 47, 1979.

Porret, M., Au lendemain de l' «Affaire Rousseau ». La «Justice pervertie» ou les représentations de la justice patricienne chez quelques publicistes de Genève, 1770-1793, Louis Binz (Éd.), Regards sur la Révolution genevoise, 1792-1798, Genève, Mémoires et documents publiés par la Société d'histoire et d'archéologie de Genève, Tome 55, 1992, pp. 122-150.

Porret, M., Effrayer le crime par la terreur des châtiments: la pédagogie de l'effroi chez quelques criminalistes du XVIII ${ }^{e}$ siècle, in Berchtold, J. et Porret, M. (Dir.), La peur au XVIII siècle, discours, représentations, pratiques, Genève, Droz, 1994, pp. 277-285.

Porret, M., Le crime et ses circonstances. De l'esprit de l'arbitraire au siècle des Lumières selon les réquisitoires des procureurs généraux de Genève, Genève, Droz, 1995.

Royer, J.-P., Histoire de la justice en France, Paris, Presses universitaires de France, 1995.

Schnapper, B., Les peines arbitraires du XIII' au XVIII ${ }^{e}$ siècle. (Doctrines savantes et usages français), Revue d'Histoire du Droit, 1973, 41, pp. 237-277 et 1974, 42, pp. 81-112.

Schnapper, B., Testes inhabiles. Les témoins reprochables dans l'ancien droit pénal, Revue d'histoire du droit, 1965, 33, pp. 575-616. 